\title{
Surface Activation of High Impact Polystyrene Substrate Using Dynamic Atmospheric Pressure Plasma
}

\author{
G. JUHÁSZ1 ${ }^{1}$ M. BERCZELI² ${ }^{2}$ Z. WELTSCH ${ }^{3}$
}

${ }^{1}$ Department of Materials Technology, GAMF Faculty of Engineering and Computer Science, John Von Neumann University, Hungary, gregoryshepard.95@gmail.com

${ }^{2}$ Department of Materials Technology, GAMF Faculty of Engineering and Computer Science, John Von Neumann University, Hungary, berczeli.miklos@gamf.uni-neumann.hu

${ }^{3}$ Department of Materials Technology, GAMF Faculty of Engineering and Computer Science, John Von Neumann University, Hungary, weltsch.zoltan@gamf.uni-neumann.hu

Abstract. Over the last decade, the number of researches has increased in the field of bonding technologies. Researchers attempt to improve surface adhesion properties by surface treatments. Adhesive bonding is one of these bonding techniques, where it is important to see what surfaces will be bonded. One such surface property is wetting, which can be improved by several types of surface treatment. In recent years, atmospheric pressure plasmas have appeared, with which research is ongoing on surface treatments. In our research, we will deal with the effects of plasma surface treatment at atmospheric pressure and its measurement. In addition, we summarize the theoretical background of adhesion, surface tension and surface treatment with atmospheric pressure plasma. Our goal is to improve adhesion properties and thus the adhesion quality.

\section{Introduction}

In today's world, the technology of adhesive bonding surrounds us everywhere. Adhesive bonding is used, for example, in space technology, automotive and everyday life. Slowly there is no household, industry, or equipment that would not apply adhesive as a bonding technique. The adhesive industry has met thousands of challenges but continues to develop [1]. The examination of adhesive bonds and the surrounding sciences had never been as important as today. The quality of adhesive bonding is attempted to improve by several researchers [7 - 9] by various surface treatments such as coating, laser surface modification, ion implantation, mechanical roughening. Over the last decade, atmospheric pressure plasmas have been introduced, which have been able to replace vacuumoperated plasma. This is of utmost importance from a practical point of view as these atmospheric plasmas can be easily integrated into the production lines [2].

The aim of our future research is to improve adhesion bonding technology with atmospheric pressure compressed air plasma treatment. 


\section{Adhesive bonding technology}

The adhesives have some basic properties such as it needs to behave as liquid at a particular stage of the bond to allow them to flow and moisten the surface, to adhere to the surface, to resist continuous and varying forces and these forces must be dissipated so that they can disperse the force between the constituents. The main essential feature of adhesives is adhesion. Adhesion is the attraction between the molecules of two different materials. This is different from cohesion, which means the attractiveness of molecules of a substance. The forces between molecules in both adhesion and cohesion are primarily Van der Waals forces. The bonds can failure either adhesively or cohesively. This means that when the bond fails adhesively, then the glue comes off from one of the two glued surfaces. If the bond is damaged cohesively, the adhesive itself will break (Figure 1). This is possible if the adhesion force is greater than the cohesive force of the adhesive or the adhesive is broken [1].
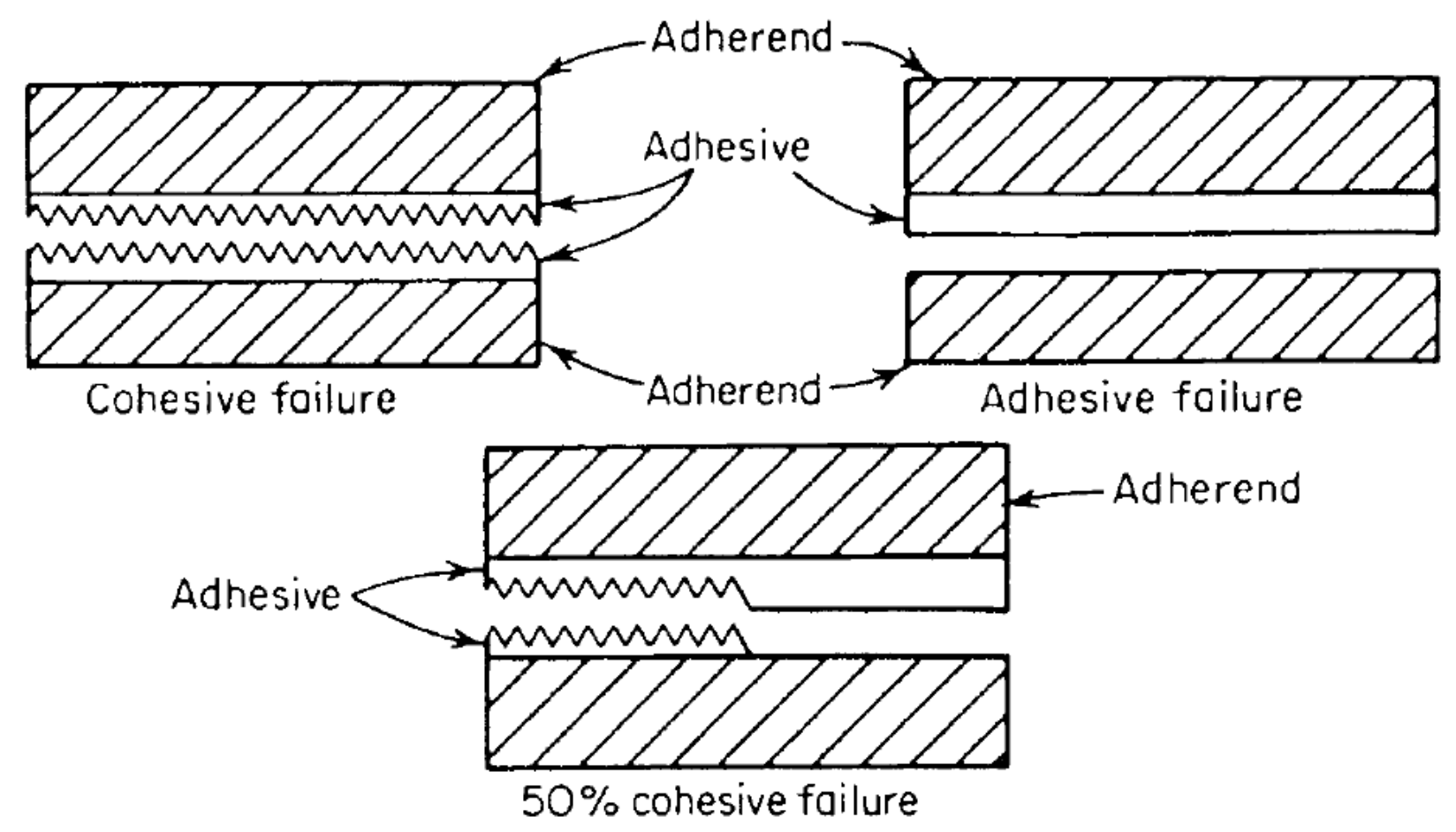

Figure 1: Examples of cohesive and adhesive failure [1]

Successful adhesive bonding also depends on several factors, such as the cleanliness of the sample to be glued, the wetting properties of the material (which is in contact with the adhesive), the solidification of the adhesive, the proper choice of the bonding arrangement. The most basic thing is to clean the surface to be glued, because if the surface is dirty (dust, oil, moisture, weak oxide layers), the adhesive will stick to these weak surface layers instead of the surface to be glued. These contaminations can be removed mechanically or chemically. The adhesive must also spread over the surface to be bonded, which is affected by wetting. If the surface is not wetted properly, then the adhesive bond will not be good quality (Figure 2) [1]. 


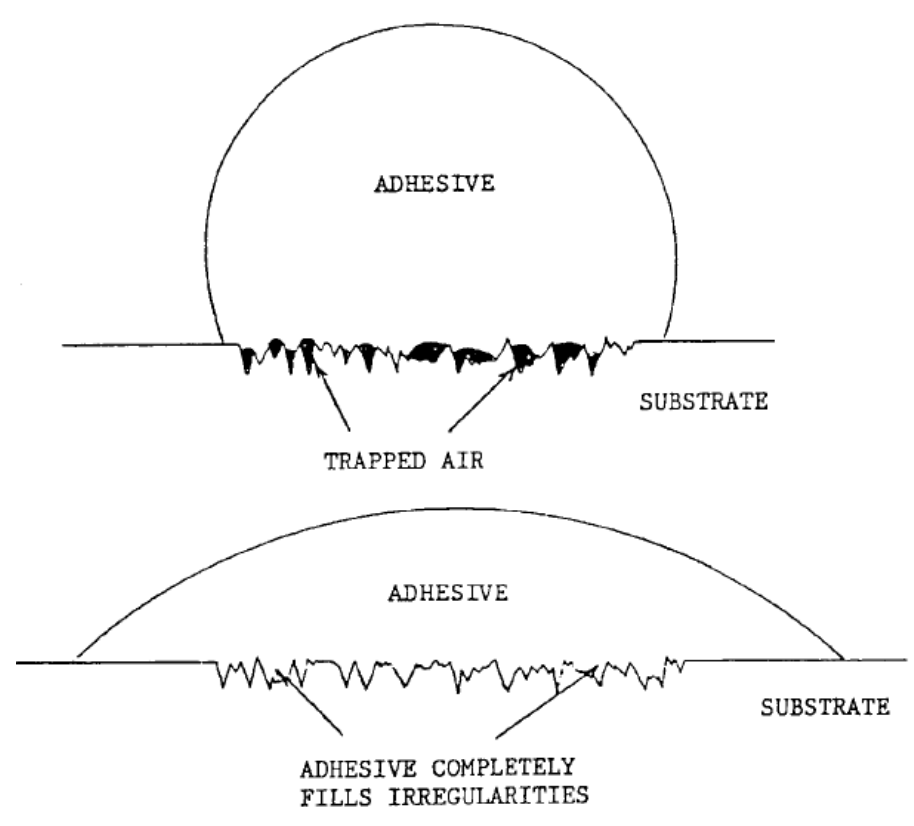

Figure 2: Illustration of bad wetting (top) and good wetting (bottom) for spreading adhesive [1]

Wetting as a definition has long been derived from everyday observations, for example, a particular fluid is not spread in the same way on every material. A cleaner surface spreads the fluid more effectively than a contaminated surface. The wetting ability can be characterized primarily by the spreading of liquids on solid surfaces [3].

\section{Surface tension}

The surface tension is closely related to the wetting concept. The surface tension is closely related to the definition of wetting. The direct measurement of wetting is the contact angle $(\Theta)$, i.e. the fit angle between the two phases. In the 19th century, Thomas Young discussed in detail the liquids' wetting conditions. The relationships described by Young are to date the basis for the description of the wetting phenomena, and the relationship created by it creates a connection between the wetting angle and the surface tension. The degree of spreading is determined by the interactions in the boundaries between the solid body and fluid molecules. The properties of the interface - thus also the wettability of the solid surfaces - which is the outermost layer are determined by especially atoms or atomic groups directly related to the liquid phase. This is called the principle of the independent surface effects, which suggests that wetting ability can be consciously influenced by the formation of appropriate structure and orientation adsorption layers [3]. 


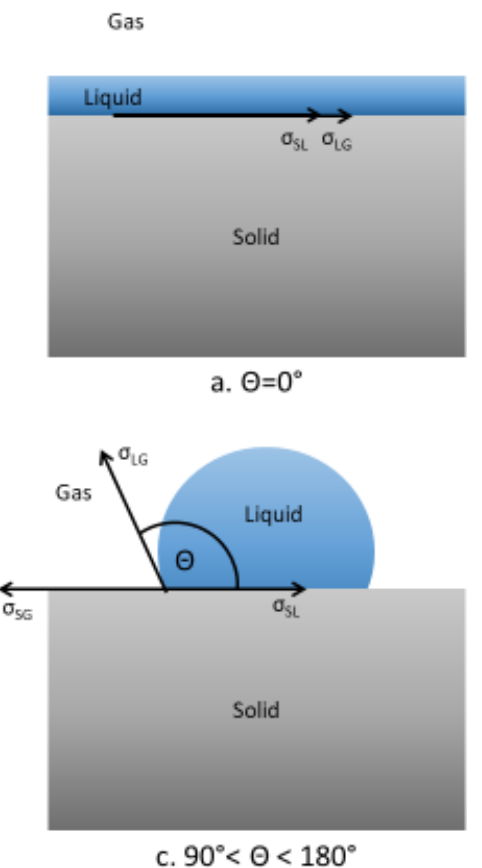

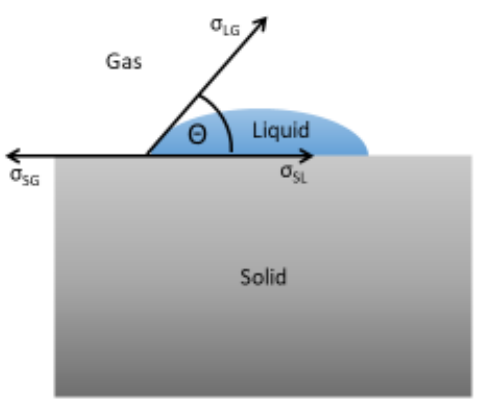

b. $0^{\circ}<\Theta<90^{\circ}$

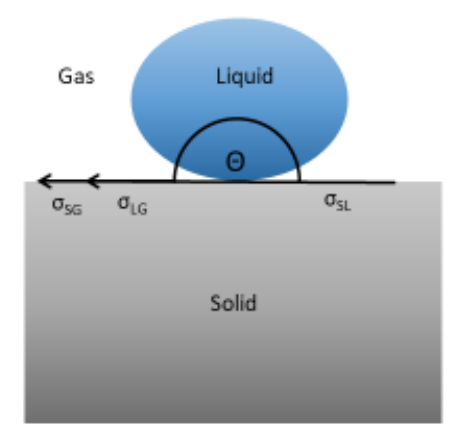

d. $\Theta=180^{\circ}$

Figure 3: The wetting angle in different cases, in the case of a liquid droplet placed on a solid phase [3]

Figure 3 shows that if the wetting angle is $0^{\circ}$, the liquid is so well spread on the surface that a liquid layer is formed, this can be due to a very good wetting properties. If this kind of contact angle is read, the surface has at least the same surface tension as the given fluid. If between $0^{\circ}$ and $90^{\circ}$, the surface is no longer hydrophilic, but if it is below $30^{\circ}$, it may still considered to be very wettable. When it is between $90^{\circ}$ and $180^{\circ}$, it is hydrophobic, the surface does not wettable enough, it can be said to be unsuitable for bonding because the adhesive tries to interact with the surrounding gas rather than the surface. If the contact angle approaches $180^{\circ}$, the surface is super-hydrophobic; in this case, the solid almost completely push away the liquid from the surface [3].

\subsection{Surface tension measurement}

Measuring the surface energy of a liquid is simple and unambiguous, since the surface energy value of a liquid equals the surface tension of the liquid. There are several techniques to measure the surface tension of a liquid. However, if we want to measure the surface energy of a solid body, then it is no longer as easy because this value is calculated by measuring the contact angle of a given number of droplets and solid bodies. Even if the proper fluids have been selected, even the surface energy of the solid body is not secure, as there are many definitions of surface energy in the literature. Therefore, there are many methods and theories that transform contact angle values into surface energy values. None of these methods are universal, as neither is perfect in reality. Therefore, they should choose fluids that meet one of these methods and have reasonable results. For non-polar surfaces it is advisable to choose non-polar liquids, while for polar solid surfaces one should choose polar liquid [4].

The most used method for determining surface energy is the Fowkes method. This method separates the surface energy of the solid body into two components, a dispersive and a polar component. 
Fowkes's method is based on three basic equations that describe interactions between solid surfaces and liquids. These three equations are:

Young's equation (1):

$$
\sigma_{S}=\sigma_{S L}+\sigma_{L} \cdot \cos \theta
$$

wherein: $\sigma_{L}$ - overall surface tension of the wetting liquid, $\sigma_{S}$ - overall surface energy of the solid $\sigma_{S L}$ the interfacial tension between the solid and the liquid, and $\theta$ - the contact angle between the liquid and the solid.

Dupre's definition of adhesion energy (2):

$$
I_{S L}=\sigma_{S}+\sigma_{L}-\sigma_{S L}
$$

wherein: $I_{S L}$ energy of adhesion per unit area between a liquid and a solid surface.

And Fowkes' theory is that the adhesion energy between the liquid and the solid surface can be divided into the interaction of the dispersion components and the interaction of polar components (3).

$$
I_{S L}=2 \cdot\left[\left(\sigma_{L}^{D}\right)^{1 / 2} \cdot\left(\sigma_{S}^{D}\right)^{1 / 2}+\left(\sigma_{L}^{P}\right)^{1 / 2} \cdot\left(\sigma_{S}{ }^{P}\right)^{1 / 2}\right]
$$

$\sigma_{L}{ }^{D}$ - dispersive component of the surface tension of the wetting liquid, $\sigma_{L}{ }^{P}$ - polar component of the surface tension of the wetting liquid, $\sigma_{S}{ }^{D}$ - dispersive component of the surface energy of the solid, $\sigma_{S}^{P}=$ polar component of the surface energy of the solid.

The combination of these three equations gives the primary equation of the Fowkes method (4):

$$
\left(\sigma_{L}^{D}\right)^{1 / 2} \cdot\left(\sigma_{S}{ }^{D}\right)^{1 / 2}+\left({\sigma_{L}}^{P}\right)^{1 / 2} \cdot\left(\sigma_{S}{ }^{P}\right)^{1 / 2}=\frac{\sigma_{L} \cdot(\cos \theta+1)}{2}
$$

Typically, the Fowkes method uses two contact angle values. A liquid with only a polar component and a liquid having a dispersive and polar component. It is recommended to use a fluid that has only a polar component because it simplifies the primary formula for the formula (5),

$$
\sigma_{S}{ }^{D}=\frac{\sigma_{L} \cdot(\cos \theta+1)^{2}}{4}
$$

with this equation dispersive component of the solid surface can be determined immediately so that the total surface tension can be calculated from this. Recommended fluids are diiodomethane and distilled water. The method can be used with two liquids with a polar and dispersive component, only one equation system must be solved because two unknowns will be in an equation. The Fowkes method can be applied to surfaces such as polyvinyl chloride, polyurethanes, polyimides, polyesters, polyacrylates, polycarbonates. As a method based on adhesion, the Fowkes method is often used for adhesion and coating problems as it can use the Fowkes equation to calculate adhesion energy between adhesive and surface or between coating and surface. The fact that, according to Fowkes, the polar component can only interact with a polar component, it predicts that the surface and adhesive will have the highest adhesion energy when the total surface tension components are similar in percent between surface and glue (Figure 4) [4]. 


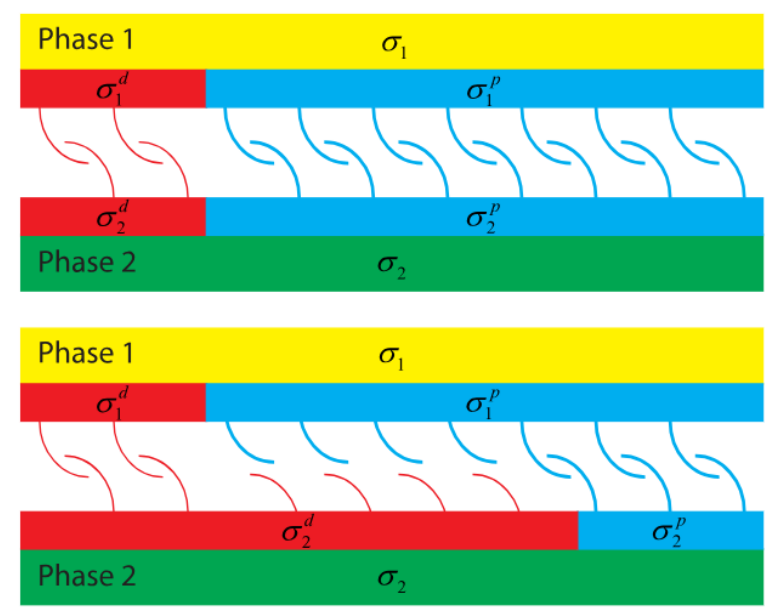

Figure 4: Illustration of the interactions between two phases with equal/similar (top) or different (bottom) dispersive and polar parts of the surface energy/tension [5]

The surface energy of the surfaces can be increased by a number of surface treatments. One such surface treatment is plasma surface treatment.

\section{Surface treatment with plasma}

Over the last fifty years, the area of plasma surface treatment increased enormously. Much of this expansion has taken place over the last decade, where there has been tremendous interest in the various techniques for preparing and modifying surfaces. Today, there are countless industrial applications, such as improving paint adhesion, bonding on the inside and surface of polymeric matrix composites, and more. The use of these plasma technologies is based on the principle that allows the surface properties of the surface of the material to be changed without altering the other properties of the material.

Atmospheric pressure plasma has practical importance for many applications. This is because, in contrast to low- or high-pressure plasma, there is no need to do the treatment in a chamber to ensure that a certain pressure is maintained for proper and consistent surface modification. Therefore, atmospheric plasma can easily be integrated into the production lines. So, you do not need the capitalintensive vacuum chambers needed to create a low pressure. Non-equilibrium atmospheric plasma applications are very wide-ranging, as functional gases can be regulated to industrial production processes and can operate from ambient temperatures up to $950^{\circ} \mathrm{C}$. In order to fulfil the functionalization requirements, voltages applied at atmospheric plasma surface functionalization are applied, ranging from $1 \mathrm{kV}$ to $20 \mathrm{kV}$. In the upper limits, the voltage generates certain wavelengths of UV radiation that contribute to free radical formation and then lead to surface activation and functionalization. By functionalizing with atmospheric plasma, it has been shown that surface wetting not only improves but also the surface free energy that has been transferred to the surface and the free energy of the treated sample can be determined. By simply using the air as a functional medium in the treatment gap, for example, it can quickly promote reactive oxygen species to promote surface oxidation of a wide range of substances [2]. 


\subsection{Surface activation}

Plasma surface treatment has two primary mechanisms for surface reactions. One is a physical reaction mechanism that is carried out with ionic activity. The other is a chemical reaction mechanism created by free radicals. During physical reactions, ionic particles get charge and kinetic energy from an electrodes electrical power supply. Molecules and atoms (and trace elements) move away from targeted surfaces as the energy of the electric field is transferred to these ions. This bombardment increases the molecular surface roughness and promotes adhesion of the interface. Plasma chemical reaction mechanisms rely on free radical effects that are generated on surfaces. These chemically active free radicals actually reduce the activation potential of the chemical reaction, which results in an atomic size removal of the surface material. These interactions can be seen in Figure 5. In general, surface reaction mechanisms are characterized by the gas-phase reaction elements on the surface of the sample [2].

1. Dissolves the surface contamination 2 . Removes the contamination from the surface

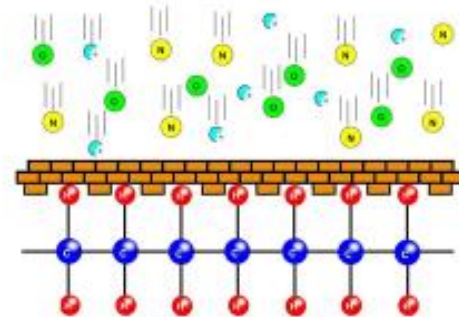

3. Activates the surface

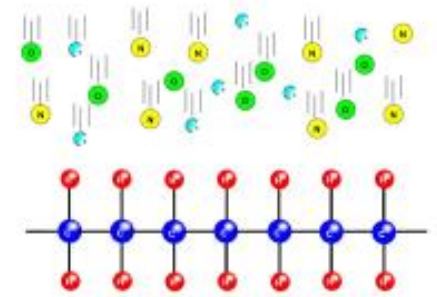

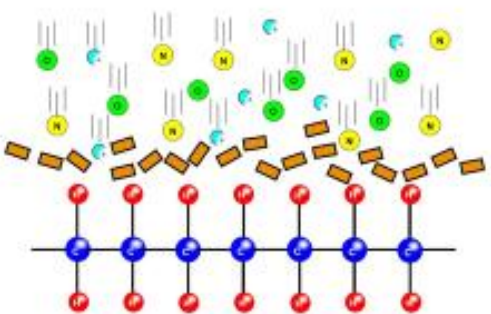

4. Functional groups are created

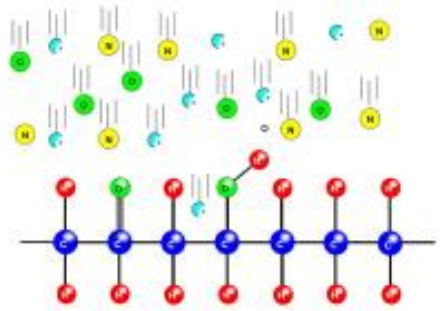

Figure 5: Effects of the plasma surface treatment

\section{Summary}

In summary, adhesive bonding is a widespread bonding technology in today's world, it is used in important places, where it is essential to create high quality bonding. Prior to gluing the surfaces have to be prepared not only for cleaning, but also to improve the wetting properties of the surfaces, because if the adhesive does not wet the surface properly, the bond quality will deteriorate. One of the properties that affects the wetting is the surface energy that can be improved by different surface treatments. One such surface treatment is atmospheric pressure plasma treatment, which is fast, efficient and environmentally friendly. It cleans and activates the surface simultaneously to aid the wetting property. 
In our future research, we will handle high impact polystyrene with atmospheric pressure plasma and adhesion experiments will be carried out on the treated surfaces.

\section{Acknowledgments}

This research was supported by EFOP-3.6.1-16-2016-000014. The Project is supported by the Hungarian Government and co-financed by the European Social Fund.

\section{References}

[1] E. M. Petrie (1999) Handbook of Adhesives and Sealants. McGraw-Hill Professional.

[2] R. A. Wolf (2013) Atmospheric Pressure Plasma for Surface Modification, Scrivener Publishing.

[3] S. Balla - K. Bán - A. Bárdos - A- Lovas - A. Szabó - Z. Weltsch (2012) Járműanyagok. Typotex Kiadó.

[4] CR, Models for Surface Free Energy Calculation, Academic research, 1999.

[5] Dispersive \& polar parts of the surface energy and surface tension Available: https://www.dataphysics-instruments.com/knowledge/understanding-interfaces/dispersivepolar-parts/ [Viewed: 2018. 07.23.]

[6] Plasmatreat surface activation knowledge, auxiliary material [Viewed: 2018. 09.03.]

[7] D. S. Patel - A. Singh - K. Balani - J. Ramkumar (2018) Topographical effects of laser surface texturing on various time-dependent wetting regimes in Ti6Al4V. Surface and Coatings Technology. 349. pp. 816-829.

[8] Z. Xu - Z. Li - S. Zhong - Z. Ma - J. Yan (2018) Wetting mechanism of Sn to Zr50.7Cu28Ni9Al12.3 bulk metallic glass assisted by ultrasonic treatment. Ultrasonics Sonochemistry. 48. pp. 207-217.

[9] Z.B. Chen - H. Bian - S.P. Hu - X.G. Song - C.N. Niu - X.K. Duan - J. Cao - J.C. Feng (2018) Surface modification on wetting and vacuum brazing behavior of graphite using AgCu filler metal. Surface and Coatings Technology. 348. pp. 104-110. 